\title{
METRICS FOR BIO-INSPIRATION BASED ON TAXONOMIES
}

\author{
Willocx, Mart (1); \\ Duflou, Joost R. (1,2) \\ 1: KU Leuven; \\ 2: Member of Flanders Make
}

\begin{abstract}
Using bio-inspiration allows engineers to use the knowledge implicitly built up by natural evolution. Current tools for providing engineers with bio-inspiration yield many biological working principles. Starting from the Linnaean taxonomy, which can be seen as a design revision history, this work proposes metrics for a working principle based on the observations of that working principle in different organisms. A first metric measures the reinforcement of a working principle via the number of observations (publications/submissions to a database) made by biologists. Furthermore, biological strategies that evolve independently and use the same working principle might be more resilient and globally applicable, prompting the proposal of a metric measuring the spread in the taxonomy. Finally, bio-novelty measures the biological novelty, inversely related to the biological diversity employing the working principle. To illustrate the use of the metrics, they are applied to the working principles identified in the 'temporary attachment' category of AskNature.
\end{abstract}

Keywords: Bio-inspired design / biomimetics, Computational design methods, Early design phases, Bio-inspiration

\section{Contact:}

Willocx, Mart

KU Leuven

Mechanical engineering

Belgium

mart.willocx@kuleuven.be 


\section{INTRODUCTION}

Even before Leonardo da Vinci's flying machines, humans have been learning from nature. Evolution has already solved many problems faced by engineers today. Due to the iterative evolution process, strategies found in nature have proven their performance (Chirazi et al., 2019). Bio-inspired design aims to use this knowledge by transferring it to engineering. Using an analogical transfer, the working principles of these strategies are applied in engineering designs (Hashemi Farzaneh and Lindemann, 2019). For example hook and loop fasteners were created after extracting the working principle of plant burrs and their ability to attach to fur (Yen et al., 2014).

A major issue of the current tools created for supporting engineers in finding relevant bio-inspiration is the number of biological strategies that are retrieved (Kaiser et al., 2013; Vandevenne et al., 2015). To alleviate this information overload, Vandevenne et al. proposed to cluster the biological strategies based on the working principle employed by the organism (Vandevenne et al., 2015).

The relevance of these working principles depends on the qualities desired by the searching engineer: they might be more interested in a proven working principle to create a robust design or desire a more novel working principle to support innovation. Reranking the retrieved bio-inspiration to take these desires into account might decrease the number of working principles that have to be evaluated during the selection \& analysis phase.

This work argues that metrics based on the taxonomy of the identified focus organisms give an indication of the performance, biological novelty and existing biological interest in the identified working principles. During a systematic search for bio-inspiration, the designer can use these metrics to quickly evaluate the possible usefulness of the retrieved working principles in view of the objectives of the design assignment. As these metrics can be automatically calculated, they can be integrated in a design support tool to rank the resulting clusters of biological documents based on the desired qualities of the working principles.

The next sections first introduce the relevant literature for systematically finding bio-inspiration, supporting filtering and selecting bio-inspiration and different forms of biological taxonomies. Second, existing methods for calculating distances in these taxonomic trees are reviewed. Third, three metrics are proposed to rank the working principle clusters based on the occurrences of their focus organisms in a taxonomy. Finally, to illustrate the use of the metrics, they are applied to the working principles identified in the case study from (Willocx et al., 2020) on 'temporary adhesion'.

\subsection{Systematically finding bio-inspiration}

To integrate bio-inspiration into systematic engineering design (engineering pull of bio-inspiration), a systematic design process has been proposed as presented in figure 1. The search for bio-inspiration starts from analysing the problem and formulating a problem description, which is used in a search operation. From the resulting biological information, the most relevant strategies are selected before they are transferred to the engineering domain (Vandevenne et al., 2015).

$\sum$ Search $\left.\left.\quad>\begin{array}{c}\text { Problem } \\ \text { formulation } \\ \text { analysis }\end{array}\right\rangle \begin{array}{c}\text { Knowledge } \\ \text { transfer }\end{array}\right\rangle$

Figure 1: The phases of the systematic bio-inspired design process as identified by Vandevenne et al. (2015).

Already several search tools have been developed which can be roughly categorized as three different approaches: direct consultation of a biologist, using a specially prepared database and using natural language biological documents for identifying bio-inspiration (Willocx et al., 2020). A major drawback of consulting an expert biologist is the limit of his or her current knowledge and the possible bias to his/her own research domain (Shu et al., 2011; Graeff et al., 2020). The major issue with database approaches is the enormous effort that is required for the population of the database with biological strategies (Vattam et al., 2011; Graeff et al., 2019a).

The natural language search tools result in a large quantity of potentially relevant biological documents, which makes the selection of relevant documents time-consuming (Kaiser et al., 2013; Willocx et al., 2020). Furthermore, as shown in (Willocx et al., 2020), due to the focus on the organism performing the strategy, many parallel strategies employing the same principle are also submitted in a database like AskNature. 
To counter this information overload, Vandevenne et al. proposed to group the retrieved documents based on the focus organism of the biological text, aiming to cluster similar biological strategies and reduce the amount of information the engineer has to navigate (Vandevenne et al., 2015). However, this does not take convergent evolution into account, where a similar working principle arises independently in two different organisms (Losos, 2011). Vandevenne et al. propose to do this clustering based on the enabling function of the biological strategy for which a method to automatically extract these is available in (Cheong and Shu, 2014) (Vandevenne et al., 2015).

\subsection{Related tools and methods for filtering and selecting bio-inspiration}

Most engineers do not have a background in biology, making filtering and understanding the retrieved biological documents a time-consuming task which can result in fixation on one biological strategy, an incorrect analogical transfer or using 'off-the-shelve' biological solutions (Helms et al., 2009; Vattam and Goel, 2011; Graeff et al., 2019b).

These pitfalls can be alleviated by including a biologist in the design team. This biologist performs a constant pre-evaluation of the biological strategies encountered, keeping only the most relevant strategies for consideration (Lenau et al., 2011). To be able to recall a large variety of relevant biological strategies, this biologist needs to have a wide basis of biology, which is a rare profile (Graeff et al., 2019a).

Tools and methods supporting filtering and selecting biological inspiration rely on having the engineer understand the retrieved strategies and manually select the most relevant for their problem (Lenau et al., 2018). Lenau et al. highlight this for the ISO approach (ISO/TC 266, 2011), the BioCards approach (Lenau, 2017) and the generic bio-inspired design method developed in (Fayemi, 2014). This reliance on the engineer to understand the biological strategy does not alleviate the time-consuming nature of filtering the retrieved bio-inspiration.

\subsection{Using taxonomies as a design history}

Evolution is an iterative process, resulting in the organisms currently roaming the earth. The genealogical history of organisms is written inside their genes. A Linnaean taxonomy aims to organize organisms with similar properties (e.g. morphological, genetic...) into coherent units, so-called taxa. These taxa are organized in a hierarchical classification with several ranks (Ruggiero et al., 2015), capturing an evolutionary history of the organisms involved. With modern molecular sequencing, a phylogenetic tree can be built based on the genetics of the organisms involved, capturing the genetic history of the organism (Woese, 2000). The genetic distance between organisms is based on the number of mutations and evolutionary events since their divergence (Nei, 2001).

Similar adaptations to taxonomically unrelated organisms can occur when the organisms occupy similar environmental contexts. This convergent evolution of the working principle is the result of natural selection of a good strategy (Losos, 2011). For example: for providing temporary attachment to diverse surfaces, AskNature contains strategies from geckos (Autumn et al., 2006), jumping spiders (Kesel, 2003), copepods (Ingram and Parker, 2006) and locusts (Han et al., 2011), all using dry adhesive pads based on the Van der Waals forces. Figure 2 locates these organisms in a Linnaean taxonomy, relating their evolutionary history.

\section{METRICS FOR BIO-INSPIRATION}

A designer is looking for effective working principles that preferably lead to innovative solutions. To guide the selection, metrics can be based on the observations of a working principle in different organisms and their positions in a taxonomic tree. First, the quantity of biological observations of a certain principle is an indication of the interest from biologists, reinforcing the value of that principle. Second, the diversity in the organisms employing the working principle is an indication for the spread of the working principle in nature. Convergent evolution might have caused different organism families to evolve an indispensable principle independently. A superior working principle is expected to spread further throughout the taxonomy. Third, a localized but large adoption rate of the principle indicates a lesser known principle, which might be useful for designers looking to innovate. The next sections detail the distance metric used in the taxonomic tree and provide a formalized definition of the proposed metrics. 


\subsection{Navigating the taxonomic tree}

Figure 2 presents the organisms in which the principle of dry adhesive pads based on the Van der Waals forces was observed in AskNature. The considered taxonomic levels are based on the four main ranks proposed in (Ruggiero et al., 2015), expanded with the rank of family, genus and species to further capture the biodiversity within an order. Taxonomic classification data is obtained from the NCBI Taxonomy database (Federhen, 2012). The observations are placed at the taxonomic level that was identified by the biologist submitting the strategy to the database or by automatic detection of the focus organism in the biological document, as proposed in (Vandevenne et al., 2014).

The taxonomic distance is defined as the length of the path traced through the classification between both species involved (Warwick and Clarke, 1995). For a Linnaean classification, as used in this research, each taxon (a group of organisms) encountered on the path, adds a constant distance. For example, the distance between the species Tokay Gecko and the phylum Chordates as illustrated in figure 2 is 5 . The distance between the species Tokay Gecko and the family Leaf Beatles is 10. Using a phylogenetic tree, the genetic distance is calculated based on the molecular differences between the different organisms (Nei, 2001). More genetically diverse organisms are located further apart.

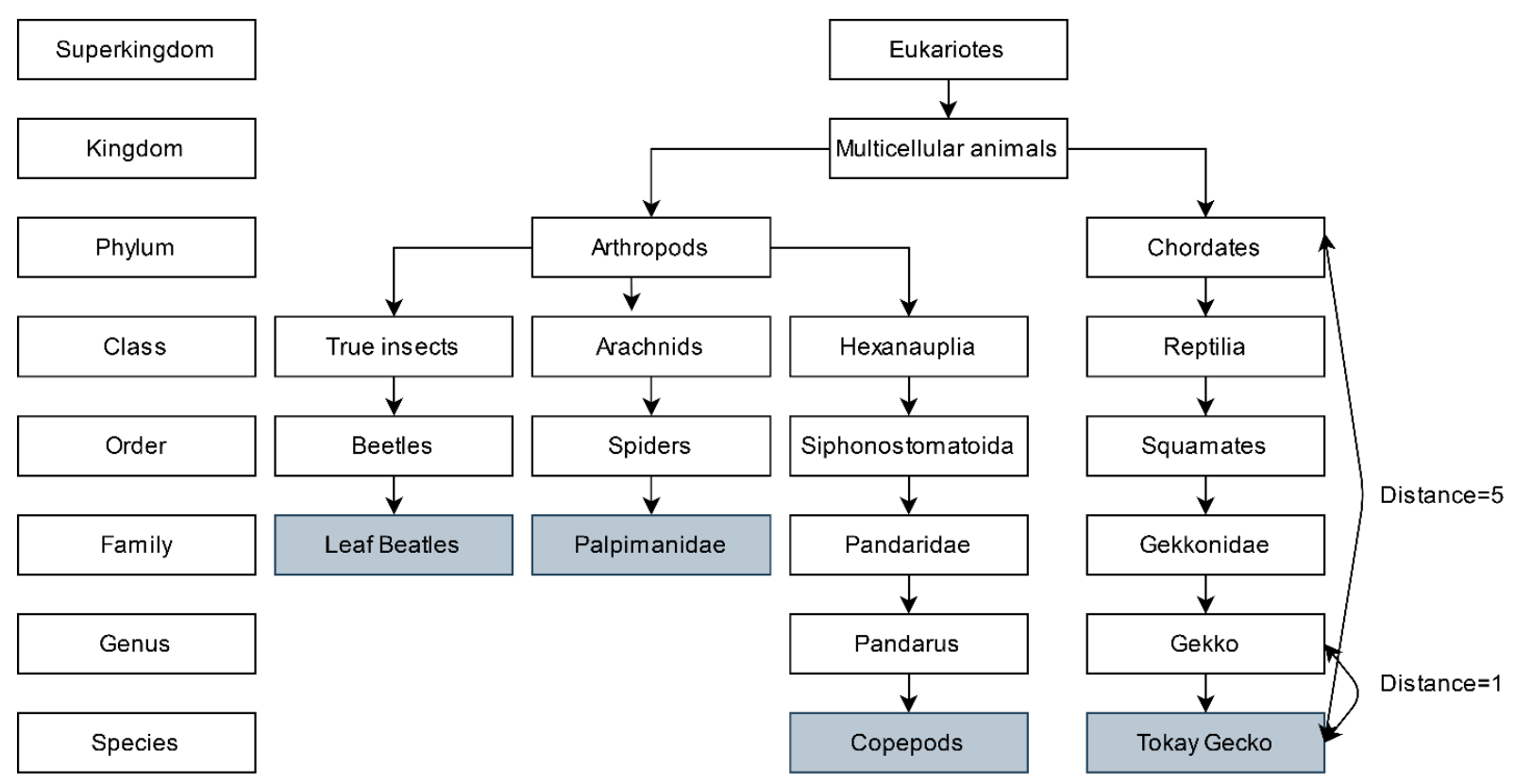

Figure 2: The taxonomic tree for the strategies in AskNature that employ dry adhesive pads based on the Van der Waals forces. Darker shaded cells indicate the level for which the observation was made. On the right, the used distance metric is demonstrated. Drawn based on the taxonomic data in the NCBI taxonomy (Federhen 2012).

\subsection{Biological interest in the working principle}

A first metric, the biological interest for the working principle is gauged by how many observations of the working principle have been made. This is a measure for the interest of the biological community for the working principle, reinforcing the belief in the working principle. Bio-interest is measured by the number of publications (natural language method) or strategies (AskNature) retrieved describing the working principle.

$$
\text { Bio-interest }=\text { number of observations of the working principle }
$$

\subsection{Spread of the working principle in the taxonomy}

A working principle that contains biological observations that have evolved in a convergent way, displays distinct clusters in the taxonomic tree. Figure 2 where the organisms in the arthropod group are located close together and further away from the Tokay gecko illustrates this. To numerically identify the working principles where this is the case, the k-medoids clustering algorithm is used to generate two or more clusters based on the distance matrix (Park and Jun, 2009). The distance between the medoids is then a measure for the distinctness of these clusters. The spread metric used here is 
based on the distance between 2-medoid clustering and is normalized by the largest distance possible. This also identifies widespread working principles in the taxonomy, as the resulting medoids will be spread out.

$$
\text { Bio-spread }=\frac{\text { distance between the 2-medoid clustering }}{14}
$$

\subsection{Biological novelty of the working principle}

A novel working principle did not spread in a large variety of organisms as defined above but does locally have a good adoption. A first indication is the locality of the strategy: most observations are close together in the taxonomy tree. Bio-novelty is defined as the inverse of the average path length between observations. This metric values working principles with local observations more. This definition results in the inverse of the taxonomic distinctness $\left(\Delta^{*}\right)$ from (Warwick and Clarke, 1995), where diversity in the number of observations made is valued more. For $n$ observations and $d_{i j}$ the taxonomic distance between observations $\mathrm{i}$ and $\mathrm{j}$ :

$$
\text { Bio-novelty }=\frac{\sum_{i}^{n} \sum_{j=i}^{n} 1}{\sum_{i}^{n} \sum_{j=i}^{n} d_{i j}+1}=\frac{1}{\Delta^{*}+1}
$$

\subsection{Use of the proposed metrics}

The aim of the metrics is to automatically rank the retrieved bio-inspiration based on the requirements of the engineer searching for bio-inspiration. The bio-spread can be used to assess the reach of adoption of the working principle and is thus a proxy for its robustness. This is useful when looking for a reliable, but not novel working principle. The bio-interest metric can be used to gauge the prior interest in a working principle. This is useful for finding lesser known working principles, for instance increasing the potential for creating novel intellectual property. The bio-novelty metric gives an indication of the novelty of the working principle in nature. Together with a low bio-interest, these metrics are a proxy for the out-of-the-box potential of the working principle. The combination of the metrics used is thus determined by the goals of the engineer and can be tuned during the session.

\subsection{Limitations}

Some limitations to the presented metrics should be pointed out. The taxonomic distance used to gauge the spread of a working principle is based on a taxonomy which still is being developed by biology researchers (Woese, 2000). Furthermore, it has been noted that some convergent evolutions are due to very similar genetic mutations, which suggests that it is the ease of making the mutation and not the superior working principle causing the convergent evolution (Stern, 2013). In addition, evolution acts on the entire organism as a system and does not guarantee an optimal working principle at sub-system level (Fish and Beneski, 2014). The manual clustering and extraction of the working principle is time-consuming and error-prone. However, with an automatic clustering and extraction of the working principle (for example as proposed in (Vandevenne et al., 2015)) these metrics can offer a quick comparison between different working principles. Finally, the NCBI taxonomy can be substituted by a phylogenetic taxonomy that yields genetic distances, which are hypothesised to be more representative for the evolutionary distance between organisms that have adopted the same working principle.

\section{APPLICATION ON BIO-INSPIRATION FOR TEMPORARY ATTACHMENT}

During a case study on the development of a mobile machining unit, bio-inspiration was retrieved for the development of a clamping interface providing temporary attachment (van Houten et al., 2021). The bio-inspiration was retrieved by consultation of an expert biologist, by searching in the AskNature database (Deldin and Schuknecht, 2014) and by using a natural language search tool (Willocx et al., 2020). The retrieved inspiration (biological articles and AskNature entries) was scanned and manually grouped by a mechanical engineer in eight different working principles: suction, mechanical friction using either a pliable skin or a claw, wet and dry adhesive pads using the Van der Waals forces, capillary adhesion and the use of polymers, either in a mucus or as strings. The resulting bioinspiration was used in the bio-based design methodology detailed in (van Houten et al., 2021). 
For the current application of the metrics, the strategies found in AskNature were tagged with their focus organism. This, together with the clustering performed in (Willocx et al., 2020) allows calculating the proposed metrics for the identified working principles. Table 1 presents the working principles and their metrics as proposed in section 2. The taxonomic distance was calculated using the ETE Toolkit (Huerta-Cepas et al., 2016) and data from the NCBI Taxonomy (Schoch et al., 2020). Strategies that combine different working principles were repeated in both relevant categories.

\section{Table 1: Metrics for the different working principles identified in the temporary attachment case study, based on the observations in the AskNature database.}

\begin{tabular}{llll} 
Working principle & Bio-interest & Spread & Bio-novelty \\
\hline Suction & 7 & 0.50 & 0.18 \\
Mechanical (skin) & 4 & 0.64 & 0.19 \\
Mechanical (claw) & 9 & 0.43 & 0.19 \\
Wet adhesive pads & 7 & 0.79 & 0.14 \\
Dry adhesive pads & 7 & 0.29 & 0.18 \\
Polymer (viscoelastic & & & \\
mucus) & 1 & 0.00 & 1.00 \\
Polymer (threads) & 9 & 0.79 & 0.12 \\
Capillary & 4 & 0.57 & 0.21
\end{tabular}

The bio-interest metric indicates that creating a temporary connection using mechanical means like a claw and the use of polymer threads are the most observed by the biological community. This is in contrast with the findings in (Willocx et al., 2020) where the contacted biologist did not identify these working principles. This might be explained by the design requirements demanding an attachment method that can attach to a large, flat surface in any direction, ruling out the use of claws, and the research focus on insect locomotion of the consulted biologist. The principles with less bio-interest have more niche applications: skin friction does not work in every orientation, a viscoelastic mucus requires a specific composition which allows it to be a combined adhesive and lubricant (Lai et al., 2010) and capillary forces' reverse dependency on the wetted area limits the force that can be transmitted.

Albeit wet and dry adhesive pads are quite similar in architecture, their measured spread in the taxonomy is very different. Dry adhesive pads require finer setae (branched tip endings) to be effective, wet adhesive pads use fluid to guarantee contact (Federle, 2006). From an engineering standpoint, producing dry adhesive pads has been difficult (Kwak et al., 2011). Despite their other drawbacks, wet adhesive pads may be easier to manufacture due to their lower tolerances. Whilst the theoretic interface strength using dry adhesive pads is higher (Federle, 2006), phylogenetic analysis has found that the selection of the used principle is based primarily in evolution and not correlated with body size (Peattie and Full, 2007). Biological research comparing the effective performance of both systems is not available (Bullock et al., 2008), but engineered dry adhesive pads outperform gecko adhesive pads (Kwak et al., 2011). Using different kinds of polymer threads to provide adhesion in a range of environments is widespread in the taxonomy and has already been used to create engineering solutions for demanding wet medical adhesion (Moulay, 2014).

The mechanical engineer scanning the relevant documents that were identified using the natural language search method, missed the most bio-novel working principles: viscoelastic mucus and capillary adhesion. This indicates that these principles were indeed unknown for the engineer, and thus their potential for inspiring designs that are more novel. Furthermore, the observation that the engineer failed to identify some of the working principles in a first pass, reinforces the need for automatic extraction and clustering of the working principles to create a scalable bio-inspired support. With automatically generated clusters, the process of calculating the metrics also does not need manual interaction, resulting in a scalable method. The principles returned by the memory search of the biologist (dry and wet adhesive pads and suction) have a lower bio-novelty score.

In conclusion, the designer looking for a working principle that has proven itself and has spread in the taxonomy, should investigate wet adhesive pads or polymer threads for attachment. In contrast, a designer looking for a less well-known principle should investigate capillary adhesion and a viscoelastic mucus, keeping the previously mentioned limitations in mind. Moreover, the metrics can 
be combined to rank the list in accordance with a (weighted) set of objectives. For instance, balancing the bio-novelty of the working principle with the bio-interest to guarantee a novel, but well-supported principle.

Finally, it should be highlighted that these findings are based on a single application of the metrics. Future research should validate the use of these metrics in different use cases for bio-inspiration based on the perception of the effectiveness and novelty of the working principle by designers.

\section{CONCLUSION}

This contribution proposed metrics for comparing different working principles identified in biological strategies based on the taxonomic tree. The bio-interest metrics allows gauging the interest from the biological community for the working principle. In combination with the spread of the working principle in the taxonomic tree, these metrics allow selecting the most widely applicable working principles from a list. The bio-novelty metric helps designers looking for innovative working principles that have proven their value but have not spread widely into the taxonomic tree. For example, principles that are useful in a niche are ranked high by this metric. Depending on the solution requirements, these metrics can be combined to rank the identified principles.

The need for automatically extracting and clustering the working principles from natural language text is reconfirmed. These metrics can be integrated into a natural language system, matching the need of the designer with a relevant working principle, shortening the time-consuming selection phase in the systematic bio-inspired design process. This clustering method will also have to take the combination of different working principles in a biological strategy into account.

\section{ACKNOWLEDGMENTS}

This work was supported by the KU Leuven Research Fund trough project GOA/15/012-SUMMA.

\section{REFERENCES}

Autumn, K., Dittmore, A., Santos, D., et al. (2006) 'Frictional adhesion: a new angle on gecko attachment', Journal of Experimental Biology, 209(18), pp. 3569-3579. https://dx.doi.org/10.1242/jeb.02486.

Bullock, J. M. R., Drechsler, P. and Federle, W. (2008) 'Comparison of smooth and hairy attachment pads in insects: friction, adhesion and mechanisms for direction-dependence', Journal of Experimental Biology, 211(20), pp. 3333-3343. https://dx.doi.org/10.1242/jeb.020941.

Cheong, H. and Shu, L. H. (2014) 'Retrieving Causally Related Functions From Natural-Language Text for Biomimetic Design', Journal of Mechanical Design, 136(8), p. 081008. https://dx.doi.org/10.1115/1.4027494.

Chirazi, J., Wanieck, K., Fayemi, P.-E., et al. (2019) 'What Do We Learn from Good Practices of Biologically Inspired Design in Innovation?', Applied Sciences, 9(4), p. 650. https://dx.doi.org/10.3390/app9040650.

Deldin, J.-M. and Schuknecht, M. (2014) 'The AskNature Database: Enabling Solutions in Biomimetic Design', in Goel, A. K., McAdams, D. A., and Stone, R. B. (eds) Biologically Inspired Design: Computational Methods and Tools. London: Springer London, pp. 17-27. https://dx.doi.org/10.1007/978-1-4471-5248-4_2.

Fayemi, P.-E. (2014) 'Bio-inspired design characterisation and its links with problem solving tools', in DS 77: Proceedings of the DESIGN 2014 13th International Design Conference. DESIGN 77, p. 10.

Federhen, S. (2012) 'The NCBI Taxonomy database', Nucleic Acids Research, 40 (Database issue), pp. D136-D143. https://dx.doi.org/10.1093/nar/gkr1178.

Federle, W. (2006) 'Why are so many adhesive pads hairy?', Journal of Experimental Biology, 209(14), pp. 2611-2621. https://dx.doi.org/10.1242/jeb.02323.

Fish, F. E. and Beneski, J. T. (2014) 'Evolution and Bio-Inspired Design: Natural Limitations', in Goel, A. K., McAdams, D. A., and Stone, R. B. (eds) Biologically Inspired Design: Computational Methods and Tools. London: Springer London, pp. 287-312. https://dx.doi.org/10.1007/978-1-4471-5248-4_12.

Graeff, E., Maranzana, N. and Aoussat, A. (2019a) 'Biomimetics, where are the biologists?', Journal of Engineering Design, 30(8-9), pp. 289-310. https://dx.doi.org/10.1080/09544828.2019.1642462.

Graeff, E., Maranzana, N. and Aoussat, A. (2019b) 'Engineers' and Biologists' Roles during Biomimetic Design Processes, Towards a Methodological Symbiosis', in Proceedings of the Design Society: International Conference on Engineering Design, pp. 319-328. https://dx.doi.org/10.1017/dsi.2019.35.

Graeff, E., Maranzana, N. and Aoussat, A. (2020) 'A shared framework of reference, a first step towards engineers' and biologists' synergic reasoning in biomimetic design teams', Journal of Mechanical Design, pp. 1-17. https://dx.doi.org/10.1115/1.4047905. 
Han, L., Wang, Z., Ji, A. and Dai, Z. (2011) 'Grip and detachment of locusts on inverted sandpaper substrates', Bioinspiration \& Biomimetics, 6(4), p. 046005. https://dx.doi.org/10.1088/1748-3182/6/4/046005.

Hashemi Farzaneh, H. and Lindemann, U. (2019) A Practical Guide to Bio-inspired Design. Berlin, Heidelberg: Springer Berlin Heidelberg. https://dx.doi.org/10.1007/978-3-662-57684-7.

Helms, M., Vattam, S. S. and Goel, A. K. (2009) 'Biologically inspired design: process and products', Design Studies, 30(5), pp. 606-622. https://dx.doi.org/10.1016/j.destud.2009.04.003.

van Houten, F., Wertheim, R., Ayali, A., et al. (2021) 'Bio-based design methodologies for products, processes, machine tools and production systems', CIRP Journal of Manufacturing Science and Technology, 32, pp. 46-60. https://dx.doi.org/10.1016/j.cirpj.2020.11.008.

Huerta-Cepas, J., Serra, F. and Bork, P. (2016) 'ETE 3: Reconstruction, Analysis, and Visualization of Phylogenomic Data', Molecular Biology and Evolution, 33(6), pp. 1635-1638. https://dx.doi.org/10.1093/molbev/msw046.

Ingram, A. L. and Parker, A. R. (2006) 'The functional morphology and attachment mechanism of pandarid adhesion pads (Crustacea: Copepoda: Pandaridae)', Zoologischer Anzeiger - A Journal of Comparative Zoology, 244(3), pp. 209-221. https://dx.doi.org/10.1016/j.jcz.2005.11.001.

ISO/TC 266 (2011) 'ISO/TC 266 - Biomimetics'. DIN.

Kaiser, M. K., Farzaneh, H. H. and Lindemann, U. (2013) 'BIOSCRABBLE: Extraction of Biological Analogies out of Large Text Sources', p. 11.

Kesel, A. B. (2003) 'Adhesion measurements on the attachment devices of the jumping spider Evarcha arcuata', Journal of Experimental Biology, 206(16), pp. 2733-2738. https://dx.doi.org/10.1242/jeb.00478.

Kwak, M. K., Pang, C., Jeong, H.-E., et al. (2011) 'Towards the Next Level of Bioinspired Dry Adhesives: New Designs and Applications', Advanced Functional Materials, 21(19), pp. 3606-3616. doi: https://doi.org/10.1002/adfm.201100982.

Lai, J. H., Alamo, J. C. del, Rodríguez-Rodríguez, J. and Lasheras, J. C. (2010) 'The mechanics of the adhesive locomotion of terrestrial gastropods', Journal of Experimental Biology, 213(22), pp. 3920-3933. https://dx.doi.org/10.1242/jeb.046706.

Lenau, T. A. (2017) 'Do biomimetic students think outside the box?', in ICED 17.

Lenau, T., Helten, K., Hepperle, C., et al. (2011) 'Reducing consequences of car collision using inspiration from nature', in Proceedings of IASDR2011: The 4th World Conference on Design Research. 4th World Conference on Design Research, Delft, Netherlands: TU Delft, p. 10.

Lenau, T., Metze, A.-L. and Hesselberg, T. (2018) 'Paradigms for biologically inspired design', in Lakhtakia, A. (ed.) Bioinspiration, Biomimetics, and Bioreplication VIII. Bioinspiration, Biomimetics, and Bioreplication VIII, Denver, United States: SPIE, p. 1. https://dx.doi.org/10.1117/12.2296560.

Losos, J. B. (2011) 'Convergence, Adaptation, and Constraint', Evolution, 65(7), pp. 1827-1840. doi: https://doi.org/10.1111/j.1558-5646.2011.01289.x.

Moulay, S. (2014) 'Dopa/Catechol-Tethered Polymers: Bioadhesives and Biomimetic Adhesive Materials', Polymer Reviews, 54(3), pp. 436-513. https://dx.doi.org/10.1080/15583724.2014.881373.

Nei, J. (2001) 'Genetic distance', in Encyclopedia of Genetics. Elsevier, pp. 828-832. https://dx.doi.org/10.1006/rwgn.2001.0532.

Park, H.-S. and Jun, C.-H. (2009) 'A simple and fast algorithm for K-medoids clustering', Expert Systems with Applications, 36(2), pp. 3336-3341. https://dx.doi.org/10.1016/j.eswa.2008.01.039.

Peattie, A. M. and Full, R. J. (2007) 'Phylogenetic analysis of the scaling of wet and dry biological fibrillar adhesives', Proceedings of the National Academy of Sciences, 104(47), pp. 18595-18600. https://dx.doi.org/10.1073/pnas.0707591104.

Ruggiero, M. A., Gordon, D. P., Orrell, T. M., et al. (2015) 'A Higher Level Classification of All Living Organisms', PLoS ONE, 10(4). https://dx.doi.org/10.1371/journal.pone.0119248.

Schoch, C. L., Ciufo, S., Domrachev, M., et al. (2020) 'NCBI Taxonomy: a comprehensive update on curation, resources and tools', Database: The Journal of Biological Databases and Curation, 2020. https://dx.doi.org/10.1093/database/baaa062.

Shu, L. H., Ueda, K., Chiu, I. and Cheong, H. (2011) 'Biologically inspired design', CIRP Annals, 60(2), pp. 673-693. https://dx.doi.org/10.1016/j.cirp.2011.06.001.

Stern, D. L. (2013) 'The genetic causes of convergent evolution', Nature Reviews Genetics, 14(11), pp. 751764. https://dx.doi.org/10.1038/nrg3483.

Vandevenne, D., Verhaegen, P.-A., Dewulf, S. and Duflou, J. R. (2015) 'SEABIRD: Scalable search for systematic biologically inspired design', Artificial Intelligence for Engineering Design, Analysis and Manufacturing, 30(01), pp. 78-95. https://dx.doi.org/10.1017/S0890060415000177.

Vandevenne, D., Verhaegen, P.-A. and Duflou, R. J. (2014) 'Mention and Focus Organism Detection and Their Applications for Scalable Systematic Bio-Ideation Tools', Journal of Mechanical Design, 136(11), p. 111104. https://dx.doi.org/10.1115/1.4028278.

Vattam, S. S. and Goel, A. K. (2011) 'Foraging for Inspiration: Understanding and Supporting the Online Information Seeking Practices of Biologically Inspired Designers', in, pp. 177-186. https://dx.doi.org/10.1115/DETC2011-48238. 
Vattam, S., Wiltgen, B., Helms, M., et al. (2011) 'DANE: Fostering Creativity in and through Biologically Inspired Design', in Taura, T. and Nagai, Y. (eds) Design Creativity 2010. London: Springer London, pp. 115-122. https://dx.doi.org/10.1007/978-0-85729-224-7_16.

Warwick, R. M. and Clarke, K. R. (1995) 'New biodiversity measures reveal a decrease in taxonomic distinctness with increasing stress', Marine Ecology Progress Series, 129, pp. 301-305. https://dx.doi.org/10.3354/meps129301.

Willocx, M., Ayali, A. and Duflou, J. R. (2020) 'Where and how to find bio-inspiration?: A comparison of search approaches for bio-inspired design', CIRP Journal of Manufacturing Science and Technology, 31, pp. 61-67. https://dx.doi.org/10.1016/j.cirpj.2020.09.013.

Woese, C. R. (2000) 'Interpreting the universal phylogenetic tree', Proceedings of the National Academy of Sciences, 97(15), pp. 8392-8396. https://dx.doi.org/10.1073/pnas.97.15.8392.

Yen, J., Helms, M., Goel, A., et al. (2014) 'Adaptive Evolution of Teaching Practices in Biologically Inspired Design', in Goel, A. K., McAdams, D. A., and Stone, R. B. (eds) Biologically Inspired Design: Computational Methods and Tools. London: Springer, pp. 153-199. https://dx.doi.org/10.1007/978-1-44715248-4_7. 


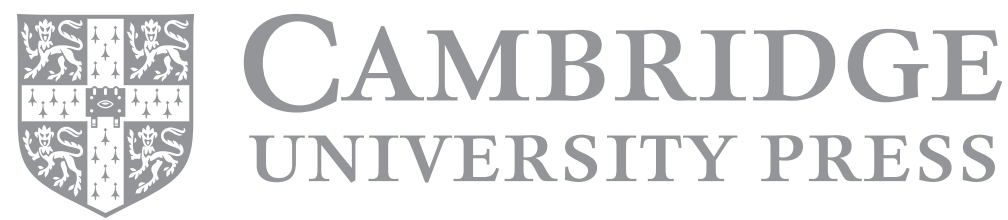

